\title{
Efficient Templated Synthesis of Donor-Acceptor Rotaxanes using Click Chemistry
}

\author{
William R. Dichtel, ${ }^{\dagger, \ddagger}$ Ognjen Š. Miljanić, ${ }^{\dagger}$ Jason M. Spruell, ${ }^{\dagger}$ \\ James R. Heath, ${ }^{\ddagger \star}$ and J. Fraser Stoddart ${ }^{\dagger *}$ \\ ${ }^{\dagger}$ California NanoSystems Institute and Department of Chemistry and Biochemistry, \\ University of California, Los Angeles, 405 Hilgard Avenue, Los Angeles, CA 90095, USA \\ ${ }^{\dagger}$ Division of Chemistry and Chemical Engineering, California Institute of Technology, \\ 1200 East California Boulevard, Pasadena, CA 91125, USA
}

\section{Supporting Information}

\begin{tabular}{|c|}
\hline Correspondence Addresses \\
\hline Professor J Fraser Stoddart \\
California NanoSystems Institute and \\
Department of Chemistry and Biochemistry \\
University of California, Los Angeles \\
405 Hilgard Avenue \\
Los Angeles, CA 90095-1569 (USA) \\
Tel: (+1)-310-206-7078 \\
Fax: (+1)-310-206-1843 \\
Email: stoddart@ chem.ucla.edu \\
Professor James R. Heath \\
of Chemistry and Chemical Engineering \\
California Institute of Technology \\
1200 East California Boulevard, \\
Pasadena, CA 91125 (USA) \\
Tel: (+1)- 626-395-6079 \\
Email: heath@ caltech.edu \\
\end{tabular}


General Methods. All reagents were purchased from commercial suppliers (Aldrich or Fisher) and used without purification. 1,5-Bis[2-(2-(2-(toluene-4sulfonyl)ethoxy)ethoxy)ethoxy]naphthalene, ${ }^{1}$ cyclobis(paraquat-p-phenylene), ${ }^{2}$ and 4 [bis[4-( $t$-butyl)phenyl][4-(isopropyl)phenyl]methyl]phenol, ${ }^{3}$ tris-1,3,5(4'-ethynylphenyl) benzene $^{4}$ were prepared using previously published procedures. Thin layer chromatography (TLC) was performed on silica gel $60 \mathrm{~F}_{254}$ (E. Merck). Preparative thin layer chromatography (Prep TLC) was performed on glass plates with a $1 \mathrm{~mm}$ thick layer of silica gel $60 \mathrm{~F}_{254}$ (E. Merck). Column chromatography was performed on silica gel $60 \mathrm{~F}$ (Merck 9385, 0.040-0.063 mm). Nuclear magnetic resonance (NMR) spectra were recorded on a Bruker Avance $600\left({ }^{1} \mathrm{H}: 600 \mathrm{MHz} ;{ }^{13} \mathrm{C}: 150 \mathrm{MHz}\right)$ or $500\left({ }^{1} \mathrm{H}: 500 \mathrm{MHz}\right.$; ${ }^{13} \mathrm{C}: 126 \mathrm{MHz}$ ) spectrometer. Chemical shifts are reported as parts per million (ppm) downfield from the $\mathrm{Me}_{4} \mathrm{Si}$ resonance as the internal standard for both ${ }^{1} \mathrm{H}$ and ${ }^{13} \mathrm{C}$ NMR spectroscopies. Electrospray ionization (ESI) mass spectra were measured on a Finnigan LCQ ion-trap mass spectrometer using $1: 1 \mathrm{MeCN}: \mathrm{H}_{2} \mathrm{O}$ as the mobile phase. Highresolution fast atom bombardment (HR-FAB) mass spectra were obtained on a JEOL JMS-600H high resolution mass spectrometer equipped with a FAB probe. Electrospray ionization mass spectra were obtained on a Finnigan LCQ ion trap mass spectrometer.

Scheme S1. Synthesis of DNP Diazide Derivative 1.

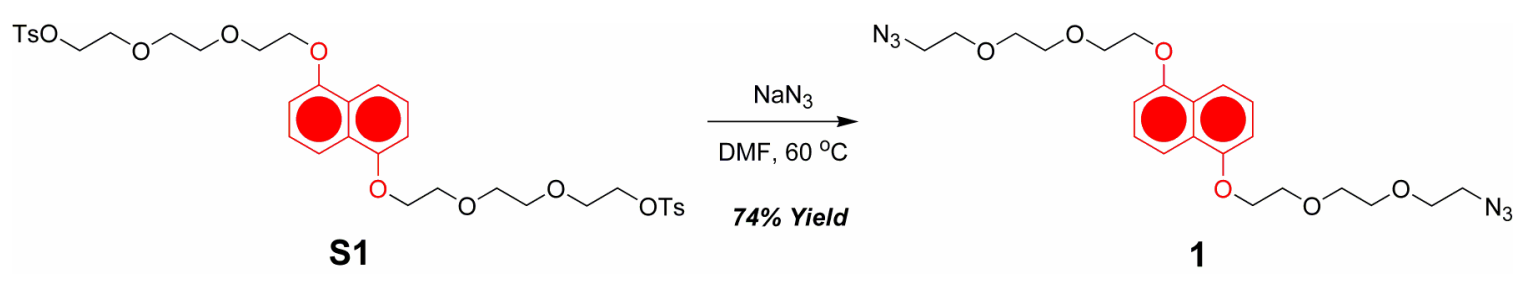

\section{Preparation of 1,5-Bis[2-(2-(2-(azide)ethoxy)ethoxy)ethoxy]naphthalene 1:}

1,5-Bis[2-(2-(2-(toluene-4-sulfonyl)ethoxy)ethoxy)ethoxy]naphthalene ${ }^{1}$ (S1，0.200 g, $0.273 \mathrm{mmol})$ and sodium azide $(0.355 \mathrm{~g}, 5.458 \mathrm{mmol})$ were dissolved in DMF (2.7 mL) and heated to $60{ }^{\circ} \mathrm{C}$ for $12 \mathrm{~h}$. The crude reaction mixture was partitioned between $100 \mathrm{~mL}$ of water and $\mathrm{CH}_{2} \mathrm{Cl}_{2}$, and the aqueous phase was washed with $\mathrm{CH}_{2} \mathrm{Cl}_{2}(3 \times 50 \mathrm{~mL})$. The combined organic extracts were washed with brine, dried $\left(\mathrm{MgSO}_{4}\right)$, and the solvent evaporated. The crude product was chromatographed $\left(\mathrm{SiO}_{2}, 7: 3 \mathrm{CH}_{2} \mathrm{Cl}_{2}: \mathrm{Et}_{2} \mathrm{O}\right.$ eluent) to 
give $96 \mathrm{mg}$ (74.4\% yield) of $\mathbf{1}$ as a pale yellow solid. 1: ${ }^{1} \mathrm{H} \mathrm{NMR}\left(500 \mathrm{MHz}, \mathrm{CDCl}_{3}, 25\right.$ ${ }^{\circ} \mathrm{C}$, TMS): $\delta 7.86\left(\mathrm{~d},{ }^{3} J(\mathrm{H}, \mathrm{H})=9 \mathrm{~Hz}, 2 \mathrm{H}, \mathrm{DNP}\right.$ aryl $\left.-\mathrm{H} p-\mathrm{O}\right), 7.35\left(\mathrm{t},{ }^{3} J(\mathrm{H}, \mathrm{H})=9 \mathrm{~Hz}\right.$, $2 \mathrm{H}, \mathrm{DNP}$ aryl $-\mathrm{H} m-\mathrm{O}), 6.84\left(\mathrm{~d},{ }^{3} \mathrm{~J}(\mathrm{H}, \mathrm{H})=8 \mathrm{~Hz}, 2 \mathrm{H}, \mathrm{DNP}\right.$ aryl $\left.-\mathrm{H} o-\mathrm{O}\right), 4.31\left(\mathrm{t},{ }^{3} J(\mathrm{H}, \mathrm{H})\right.$ $\left.=5 \mathrm{~Hz}, 4 \mathrm{H}, \mathrm{DNP}-\mathrm{OCH}_{2}\right), 4.00\left(\mathrm{t},{ }^{3} \mathrm{~J}(\mathrm{H}, \mathrm{H})=5 \mathrm{~Hz}, 4 \mathrm{H}\right), 3.82\left(\mathrm{t},{ }^{3} \mathrm{~J}(\mathrm{H}, \mathrm{H})=9 \mathrm{~Hz}, 4 \mathrm{H}\right)$, $3.71(\mathrm{~m}, 8 \mathrm{H}), 3.37\left(\mathrm{t},{ }^{3} \mathrm{~J}(\mathrm{H}, \mathrm{H})=5 \mathrm{~Hz}, 4 \mathrm{H}, \mathrm{CH}_{2} \mathrm{~N}_{3}\right) ;{ }^{13} \mathrm{C} \mathrm{NMR}\left(125 \mathrm{MHz}, \mathrm{CDCl}_{3}, 25{ }^{\circ} \mathrm{C}\right)$ : $\delta$ 154.2, 126.7, 125.0, 114.5, 105.6, 71.0, 70.7, 70.0, 69.8, 67.8, 50.6; HRMS (FAB): Calcd for $\mathrm{C}_{22} \mathrm{H}_{30} \mathrm{~N}_{6} \mathrm{O}_{6} \mathrm{~m} / z=474.2227$. Found $m / z=474.2226$.

Scheme S2. Synthesis of Alkyne Functionalized Stopper 2.

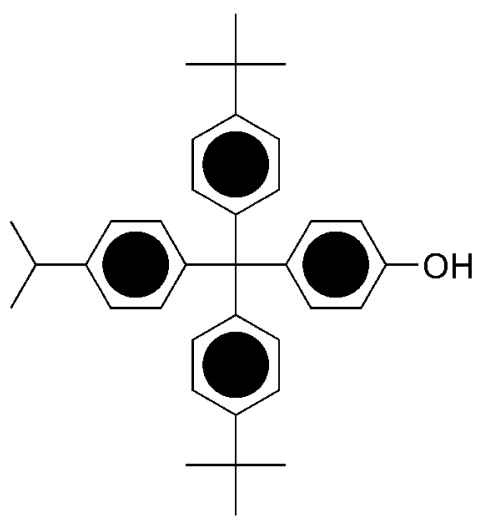

S2

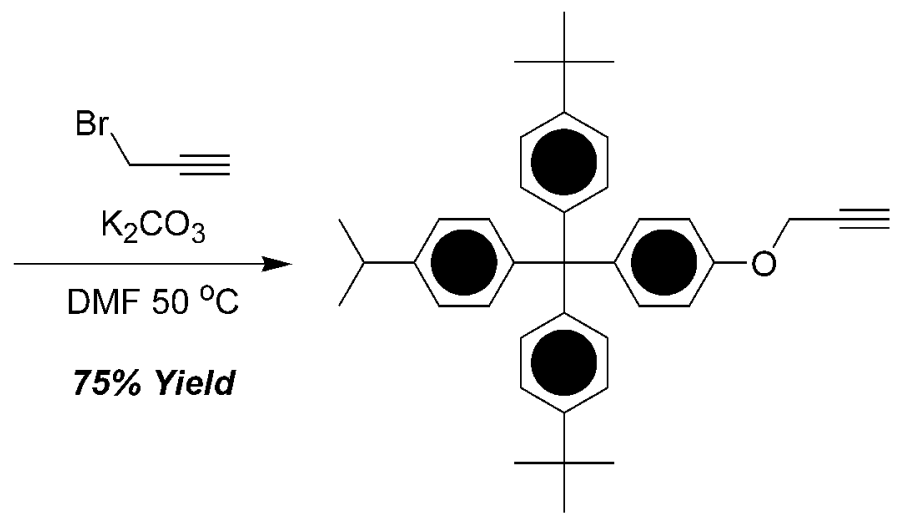

2

\section{Preparation of 4-[bis[4-(t-butyl)phenyl][4-(isopropyl)phenyl]methyl]phenyl propargyl ether (2):}

Tetraarylmethane $\mathbf{S 2}$ (4-[bis[4-(t-butyl)phenyl][4-(isopropyl)phenyl]methyl]phenol, ${ }^{3}$ $0.750 \mathrm{~g}, 1.528 \mathrm{mmol})$ and $\mathrm{K}_{2} \mathrm{CO}_{3}(0.634 \mathrm{~g}, 4.585 \mathrm{mmol})$ were suspended in DMF (7.6 $\mathrm{mL}$ ). Propargyl bromide ( $0.6 \mathrm{~g}$ of an $80 \mathrm{wt} \%$ solution in xylenes, $3.47 \mathrm{mmol}$ ) was added and the solution was heated to $50{ }^{\circ} \mathrm{C}$ for $12 \mathrm{~h}$. The reaction mixture was poured in $75 \mathrm{~mL}$ of EtOAc and was washed with $1 \mathrm{M} \mathrm{NaHSO}_{4}(75 \mathrm{~mL}), \mathrm{H}_{2} \mathrm{O}(2 \times 75 \mathrm{~mL})$, brine $(75 \mathrm{~mL})$, and dried $\left(\mathrm{MgSO}_{4}\right)$. The solution was evaporated onto silica gel, loaded onto a silica gel column, and chromatographed using 1:9 EtOAc:hexanes as the eluent to obtain $0.602 \mathrm{~g}$ (75\% yield) of 2 as a white solid. 2: ${ }^{1} \mathrm{H}$ NMR (500 MHz, $\mathrm{CDCl}_{3}, 25{ }^{\circ} \mathrm{C}$, TMS): 7.22 (d, ${ }^{2} J(\mathrm{H}, \mathrm{H})=9 \mathrm{~Hz}, 4 \mathrm{H}, \mathrm{Ar}-\mathrm{H}$ ortho-tBu $), 7.12\left(\mathrm{~d},{ }^{2} J(\mathrm{H}, \mathrm{H})=7 \mathrm{~Hz}, 2 \mathrm{H}, \mathrm{Ar}-\mathrm{H}, \mathrm{Ar}-\mathrm{H}\right.$ orthoiPr), $7.07\left(\mathrm{~m}, 8 \mathrm{H}\right.$, all meta Ar-H), $6.85\left(\mathrm{~d},{ }^{2} J(\mathrm{H}, \mathrm{H})=6 \mathrm{~Hz}, 2 \mathrm{H}, \mathrm{Ar}-\mathrm{H}\right.$ ortho-O $), 4.66(\mathrm{~d}$, $\left.{ }^{2} J(\mathrm{H}, \mathrm{H})=2 \mathrm{~Hz},-\mathrm{O}-\mathrm{CH}_{2}-\right), 2.88\left(\right.$ septet, $\left.^{2} J(\mathrm{H}, \mathrm{H})=7 \mathrm{~Hz}, 1 \mathrm{H},-\mathrm{CH}\left(\mathrm{CH}_{3}\right)_{2}\right), 2.52\left(\mathrm{t},{ }^{2} J(\mathrm{H}, \mathrm{H})\right.$ 
$=2 \mathrm{~Hz}, 1 \mathrm{H}, \mathrm{CCH}), 1.30(\mathrm{~s}, 18 \mathrm{H}, \mathrm{tBu}), 1.24\left(\mathrm{~d},{ }^{2} \mathrm{~J}(\mathrm{H}, \mathrm{H})=7 \mathrm{~Hz}, 6 \mathrm{H}, \mathrm{iPr}\right) ;{ }^{13} \mathrm{C}$ NMR $(125$ $\left.\mathrm{MHz}, \mathrm{CDCl}_{3}\right): \delta 155.4,148.2,146.0,144.4,144.0,140.4,132.2,130.9,130.6,125.1$, 124.0, 113.2, 78.7, 75.3, 63.1, 55.7, 34.2, 33.4, 31.3, 23.9; HRMS (FAB): Calcd for $\mathrm{C}_{39} \mathrm{H}_{45} \mathrm{O} \mathrm{m} / z=529.3470$. Found $\mathrm{m} / z=529.3449$.

\section{Preparation of the $[2]$ Rotaxane $3 \cdot 4 \mathrm{PF}_{6}$ :}

Diazide DNP derivative $1(0.0070 \mathrm{~g}, 0.015 \mathrm{mmol}), \mathrm{CBPQT}^{4+}(0.017 \mathrm{~g}, 0.015 \mathrm{mmol})$, and the alkyne functionalized stopper $2(0.016 \mathrm{~g}, 0.031 \mathrm{mmol})$ were dissolved in DMF $(0.160$ $\mathrm{mL}$ ) at $-10{ }^{\circ} \mathrm{C}$, forming a deep red solution. Stock solutions of $\mathrm{CuSO}_{4} \cdot 5 \mathrm{H}_{2} \mathrm{O}$ in DMF $(20 \mu \mathrm{L}, 0.074 \mathrm{M})$ and ascorbic acid in DMF $(20 \mu \mathrm{L}, 0.148 \mathrm{M})$ were added. The solution was stirred at $-10{ }^{\circ} \mathrm{C}$ for $24 \mathrm{~h}$, at which time the solvent was evaporated. The red solid was redissolved in $\mathrm{Me}_{2} \mathrm{CO}$ and the [2] rotaxane was purified by preparative TLC using a $1 \% \mathrm{w} / \mathrm{v} \mathrm{NH}_{4} \mathrm{PF}_{6}$ solution in $\mathrm{Me}_{2} \mathrm{CO}$ as the mobile phase. The rotaxane product was recovered from the silica gel by washing with an excess of eluent solution. The $\mathrm{Me}_{2} \mathrm{CO}$ was concentrated to a minimum volume, and the product was precipitated from this solution through the addition of an excess of cold water. The click [2] rotaxane $3 \cdot 4 \mathrm{PF}_{6}$ was isolated as a purple solid $\left(0.033 \mathrm{~g}, 84 \%\right.$ yield). $3 \cdot 4 \mathrm{PF}_{6}:{ }^{1} \mathrm{H}$ NMR $(500 \mathrm{MHz}$, $\mathrm{CD}_{3} \mathrm{COCD}_{3}, 52{ }^{\circ} \mathrm{C}$ ): $\delta 9.21$ (br s, $8 \mathrm{H}, \alpha-\mathrm{CBPQT}^{4+}$ ), $8.29\left(\mathrm{~s}, 8 \mathrm{H}, \beta-\mathrm{CBPQT}^{4+}\right), 8.02$ (s, $2 \mathrm{H}$, triazole-H), $7.75\left(\right.$ br s, 8H, aryl-CBPQT $\left.{ }^{4+}\right), 7.29\left(\mathrm{~d},{ }^{3} \mathrm{~J}(\mathrm{H}, \mathrm{H})=7 \mathrm{~Hz}, 8 \mathrm{H}\right.$, stopper aryl-H $o$-tBu $), 7.11(\mathrm{~m}, 20 \mathrm{H}$, stopper - all meta Ar-H plus $o$-iPr aryl $-\mathrm{H}), 6.84\left(\mathrm{~d},{ }^{3} J(\mathrm{H}, \mathrm{H})\right.$ $=9 \mathrm{~Hz}, 4 \mathrm{H}$, stopper aryl $-\mathrm{H} o-\mathrm{O}), 6.49\left(\mathrm{~d},{ }^{3} J(\mathrm{H}, \mathrm{H})=8 \mathrm{~Hz}, 2 \mathrm{H}, \mathrm{DNP} o-\mathrm{O}\right), 6.26\left(\mathrm{t},{ }^{3} J\right.$ $(\mathrm{H}, \mathrm{H})=8 \mathrm{~Hz}, 2 \mathrm{H}, \mathrm{DNP}-m-\mathrm{O}), 6.02\left(\mathrm{br} \mathrm{s}, 8 \mathrm{H}, \mathrm{CBPQT}^{4+}\right.$ benzyl H), 4.97 (s, 4H, stopper$\left.\mathrm{OCH}_{2}\right), 4.61\left(\mathrm{t},{ }^{3} \mathrm{~J}(\mathrm{H}, \mathrm{H})=5 \mathrm{~Hz}, 4 \mathrm{H}\right), 4.50(\mathrm{br} \mathrm{m}, 4 \mathrm{H}), 4.30(\mathrm{br} \mathrm{m}, 4 \mathrm{H}), 4.15\left(\mathrm{t},{ }^{3} \mathrm{~J}(\mathrm{H}, \mathrm{H})\right.$ $=5 \mathrm{~Hz}, 4 \mathrm{H}), 4.09$ (br m, 4H), 4.00 (br m, 4H), 2.89 (septet, ${ }^{3} \mathrm{~J}(\mathrm{H}, \mathrm{H})=7 \mathrm{~Hz}, 2 \mathrm{H}$, stopper iPr H), $2.80\left(\mathrm{~d},{ }^{3} J(\mathrm{H}, \mathrm{H})=8 \mathrm{~Hz}, 2 \mathrm{H}, \mathrm{DNP} p-\mathrm{O}\right), 1.31(\mathrm{~s}, 36 \mathrm{H}, \mathrm{tBu}), 1.23\left(\mathrm{~d},{ }^{3} J(\mathrm{H}, \mathrm{H})=7\right.$ $\left.\mathrm{Hz}, 12 \mathrm{H}, \mathrm{iPr} \mathrm{CH}_{3}\right) ;{ }^{13} \mathrm{C} \mathrm{NMR}\left(125 \mathrm{MHz}, \mathrm{CD}_{3} \mathrm{COCD}_{3}, 52{ }^{\circ} \mathrm{C}\right): 149.6,147.3,146.7,145.8$, 145.4, 137.9, 133.1, 132.6, 131.9, 131.7, 129.3, 126.3, 125.8, 125.2, 114.6, 110.0, 105.9, 72.1, 71.8, 71.1, 70.5, 69.3, 66.6, 64.3, 62.6, 35.0, 34.3, 31.8, 24.3; MS (ESI, 1:1 $\left.\mathrm{MeCN}: \mathrm{H}_{2} \mathrm{O}, 0.1 \% \mathrm{AcOH}\right)$ : Found $1170.9\left(M-2 \mathrm{PF}_{6}\right)^{2+}, 723.3\left(M-3 \mathrm{PF}_{6}\right)^{3+}, 512.9(M-$ $\left.4 \mathrm{PF}_{6}\right)^{4+}$. 
General Click Chemistry Procedure for the Synthesis of High Order Rotaxanes and Preparation of [3]Rotaxane $6 \cdot 8 \mathrm{PF}_{6}$ :

DNP derivative 4 (0.013 g, $0.0155 \mathrm{mmol}, 1.05$ eq. per alkyne), CBPQT ${ }^{4+}(0.0195 \mathrm{~g}$, $0.0178 \mathrm{mmol}, 1.2$ eq. per alkyne), and the dialkyne functionalized tetraarylmethane $\mathbf{5}$ $(0.0040 \mathrm{~g}, 0.0074 \mathrm{mmol})$ were dissolved in DMF $(0.090 \mathrm{~mL})$ at $-10{ }^{\circ} \mathrm{C}$, forming a deep red solution. Stock solutions of $\mathrm{CuSO}_{4} \cdot 5 \mathrm{H}_{2} \mathrm{O}$ in DMF $(20 \mu \mathrm{L}, 0.036 \mathrm{M}, 0.05$ eq per alkyne) and ascorbic acid in DMF ( $20 \mu \mathrm{L}, 0.072 \mathrm{M}, 0.1$ eq. per alkyne) were added. The solution was stirred at $-10{ }^{\circ} \mathrm{C}$ for $24 \mathrm{~h}$, at which time the solvent was evaporated. The red solid was redissolved in $\mathrm{Me}_{2} \mathrm{CO}$ and the product [2] rotaxane was purified by preparative TLC using a $2 \% \mathrm{w} / \mathrm{v} \mathrm{NH}_{4} \mathrm{PF}_{6}$ solution in $\mathrm{Me}_{2} \mathrm{CO}$ as the mobile phase. The rotaxane product was recovered from the silica gel by washing with an excess of $\mathrm{Me}_{2} \mathrm{CO}$ / $\mathrm{NH}_{4} \mathrm{PF}_{6}$ solution. The $\mathrm{Me}_{2} \mathrm{CO}$ was concentrated to a minimum volume, and the product was precipitated from this solution through the addition of an excess of cold water. The click [3] rotaxane $6 \cdot 8 \mathrm{PF}_{6}(0.033 \mathrm{~g}, 84 \%$ yield $)$ was isolated by filtration as a purple solid and dried under high vacuum overnight. $6 \cdot 8 \mathrm{PF}_{6}:{ }^{1} \mathrm{H} \mathrm{NMR}\left(500 \mathrm{MHz}, \mathrm{CD}_{3} \mathrm{CN}, 75{ }^{\circ} \mathrm{C}\right): \delta$ 8.77 (br s, 16H, $\alpha$-CBPQT ${ }^{4+}$ ), 8.07 (s, 2H, triazole-H), 7.99 (s, 16H, $\beta-\mathrm{CBPQT}^{4+}$ ), 7.30 $\left(\mathrm{m}, 28 \mathrm{H}\right.$, aryl-CBPQT ${ }^{4+}$, all stopper aryl-H $\left.o-\mathrm{tBu}\right), 7.10(\mathrm{~m}, 28 \mathrm{H}$, stopper $\mathrm{m}-$ aryl $-\mathrm{H}$, stopper aryl -H o-iPr), $6.86(\mathrm{~m}, 8 \mathrm{H}$, stopper aryl $-\mathrm{H} o-\mathrm{O}), 6.41\left(\mathrm{~d},{ }^{3} \mathrm{~J}(\mathrm{H}, \mathrm{H})=8 \mathrm{~Hz}, 2 \mathrm{H}\right.$, DNP $o-\mathrm{O}), 6.37\left(\mathrm{~d},{ }^{3} J(\mathrm{H}, \mathrm{H})=8 \mathrm{~Hz}, 2 \mathrm{H}, \mathrm{DNP} o-\mathrm{O}\right), 6.01(\mathrm{~m}, 4 \mathrm{H}, \mathrm{DNP}-m-\mathrm{O}), 5.79\left(\mathrm{~d},{ }^{2} J\right.$ $(\mathrm{H}, \mathrm{H})=13 \mathrm{~Hz}, 8 \mathrm{H}, \mathrm{CBPQT}^{4+}$ benzyl H$), 5.79\left(\mathrm{~d},{ }^{2} J(\mathrm{H}, \mathrm{H})=13 \mathrm{~Hz}, 8 \mathrm{H}, \mathrm{CBPQT}^{4+}\right.$ benzyl $\mathrm{H}), 5.11\left(\mathrm{~s}, 4 \mathrm{H}, \mathrm{OCH}_{2}\right.$-triazole), $4.83\left(\mathrm{t},{ }^{3} \mathrm{~J}(\mathrm{H}, \mathrm{H})=5 \mathrm{~Hz}, 4 \mathrm{H}\right.$, triazole $\left.-\mathrm{CH}_{2}\right), 4.46(\mathrm{br} \mathrm{m}$, $8 \mathrm{H}), 4.30(\mathrm{br} \mathrm{m}, 12 \mathrm{H}), 4.25(\mathrm{~m}, 8 \mathrm{H}), 2.88$ (septet, ${ }^{3} \mathrm{~J}(\mathrm{H}, \mathrm{H})=7 \mathrm{~Hz}, 2 \mathrm{H}$, stopper iPr $\mathrm{H}$ ), $2.56\left(\mathrm{~d},{ }^{3} J(\mathrm{H}, \mathrm{H})=8 \mathrm{~Hz}, 2 \mathrm{H}, \mathrm{DNP} p-\mathrm{O}\right), 2.55\left(\mathrm{~d},{ }^{3} J(\mathrm{H}, \mathrm{H})=8 \mathrm{~Hz}, 2 \mathrm{H}, \mathrm{DNP} p-\mathrm{O}\right), 1.31$ (s, $18 \mathrm{H}$, central tBu), $1.29(\mathrm{~s}, 36 \mathrm{H}$, external $\mathrm{tBu}), 1.22\left(\mathrm{~d},{ }^{3} J(\mathrm{H}, \mathrm{H})=7 \mathrm{~Hz}, 12 \mathrm{H}\right.$, stopper iPr); ${ }^{13} \mathrm{C}$ MS (ESI, $\left.1: 1 \mathrm{MeCN}: \mathrm{H}_{2} \mathrm{O}, 0.1 \% \mathrm{AcOH}\right): \delta 156.2,156.1,150.9,148.5,148.4$, 146.2, 145.2, 144.7, 144.3, 144.3, 143.8, 140.1, 140.0, 136.4, 131.8, 131.6, 131.2, 130.3, 130.0, 128.0, 127.9, $126.1125 .4,124.6,124.3,124.2,124.2,113.5,113.4,108.2,104.4$, 104.3, 70.4, 69.8, 69.6, 68.2, 68.0, 65.0, 62.9, 62.6, 61.1, 50.5, 33.8, 33.8, 33.1, 30.5, 30.4, 23.1; Found $1324.6\left(M-3 \mathrm{PF}_{6}\right)^{3+}, 957.3\left(M-4 \mathrm{PF}_{6}\right)^{4+}, 736.9\left(M-5 \mathrm{PF}_{6}\right)^{5+}, 589.9(M$ $\left.-6 \mathrm{PF}_{6}\right)^{6+}, 484.9\left(M-7 \mathrm{PF}_{6}\right)^{7+}$. 


\section{Preparation of [4]Rotaxane $8 \cdot \mathbf{1 2 P F}_{6}$ :}

The above procedure was followed using tris-1,3,5(4'-ethynylphenyl)benzene $\mathbf{7}^{5}(0.0040$ $\mathrm{g}, 0.011 \mathrm{mmol})$ as the alkyne containing component. Preparative TLC as described above provided the [4] rotaxane $\mathbf{8} \cdot 12 \mathrm{PF}_{6}(47.5 \mathrm{mg}, 72 \%$ yield $)$ as a purple solid. $\mathbf{8}$. 12PF 6 : ${ }^{1} \mathrm{H}$ NMR $\left(500 \mathrm{MHz}, \mathrm{CD}_{3} \mathrm{COCD}_{3}, 52{ }^{\circ} \mathrm{C}\right): \delta 9.17\left(\mathrm{br} \mathrm{s}, 24 \mathrm{H}, \alpha-\mathrm{CBPQT}^{4+}\right), 8.61(\mathrm{~s}$, $3 \mathrm{H}$, triazole-H), $8.28\left(\mathrm{~s}, 24 \mathrm{H}, \beta-\mathrm{CBPQT}^{4+}\right), 7.79\left(\mathrm{~m}, 39 \mathrm{H}\right.$, aryl-CBPQT ${ }^{4+}$, all central unit Ar-H), $7.26\left(\mathrm{~d},{ }^{3} J(\mathrm{H}, \mathrm{H})=7 \mathrm{~Hz}, 12 \mathrm{H}\right.$, stopper aryl $-\mathrm{H} o$-tBu), 7.07 (m, 30H, stopper - all meta Ar-H plus $o$-iPr aryl -H), $6.88\left(\mathrm{~d},{ }^{3} J(\mathrm{H}, \mathrm{H})=9 \mathrm{~Hz}, 6 \mathrm{H}\right.$, stopper aryl $\left.-\mathrm{H} o-\mathrm{O}\right), 6.49$ $\left(\mathrm{d},{ }^{3} \mathrm{~J}(\mathrm{H}, \mathrm{H})=8 \mathrm{~Hz}, 6 \mathrm{H}, \mathrm{DNP} o-\mathrm{O}\right), 6.22$ (overlapping $\mathrm{t},{ }^{3} \mathrm{~J}(\mathrm{H}, \mathrm{H})=8 \mathrm{~Hz}, 6 \mathrm{H}$, both DNP$m$-O), 6.03 (br m, 24H, CBPQT ${ }^{4+}$ benzyl H), $4.00\left(\mathrm{t},{ }^{3} \mathrm{~J}(\mathrm{H}, \mathrm{H})=8 \mathrm{~Hz}, 6 \mathrm{H}\right.$, stopper$\left.\mathrm{OCH}_{2}\right), 4.55(\mathrm{~m}, 12 \mathrm{H}), 4.50(\mathrm{~m}, 12 \mathrm{H}), 4.43(\mathrm{~m}, 12 \mathrm{H}), 4.32\left(\mathrm{t},{ }^{3} \mathrm{~J}(\mathrm{H}, \mathrm{H})=5 \mathrm{~Hz}, 6 \mathrm{H}\right.$, triazole- $\mathrm{NCH}_{2}$ ), 2.86 (septet, ${ }^{3} J(\mathrm{H}, \mathrm{H})=7 \mathrm{~Hz}, 3 \mathrm{H}$, stopper $\left.\mathrm{iPr} \mathrm{H}\right), 2.55(\mathrm{br} \mathrm{s}, 3 \mathrm{H}, \mathrm{DNP} p$ O), $1.28(\mathrm{~s}, 54 \mathrm{H}, \mathrm{tBu}), 1.20\left(\mathrm{~d},{ }^{3} \mathrm{~J}(\mathrm{H}, \mathrm{H})=7 \mathrm{~Hz}, 12 \mathrm{H}, \mathrm{iPr} \mathrm{CH}_{3}\right) ;{ }^{13} \mathrm{C}$ NMR $(125 \mathrm{MHz}$, $\left.\mathrm{CD}_{3} \mathrm{COCD}_{3}, 2{ }^{\circ} \mathrm{C}\right): 156.2,150.8,148.4,147.2,146.2,145.2,144.7,144.3,143.6,141.4$, 140.2, 140.0, 136.3, 131.8, 131.2, 130.3, 130.0, 127.9, 127.9, 127.7, 126.2, 125.7, 125.4, 124.6, 124.3, 124.2, 121.5, 113.4, 108.2, 104.4, 104.2, 70.35, 69.9, 69.7, 68.1, 68.0, 65.0, 62.9,50.5, 33.8, 33.1, 30.4, 23.1; MS (ESI, $1: 1 \mathrm{MeCN}: \mathrm{H}_{2} \mathrm{O}, 0.1 \%$ AcOH): Found 1915.2 $\left(M-3 \mathrm{PF}_{6}\right)^{3+}, 1400.3\left(M-4 \mathrm{PF}_{6}\right)^{4+}, 1091.3\left(M-5 \mathrm{PF}_{6}\right)^{5+}, 885.3\left(M-6 \mathrm{PF}_{6}\right)^{6+}, 738.1(M-$ $\left.7 \mathrm{PF}_{6}\right)^{7+}, 627.7\left(M-8 \mathrm{PF}_{6}\right)^{8+}, 541.8\left(M-9 \mathrm{PF}_{6}\right)^{9+}$.

Scheme S3. Preparation of DNP Azide Derivative 4.

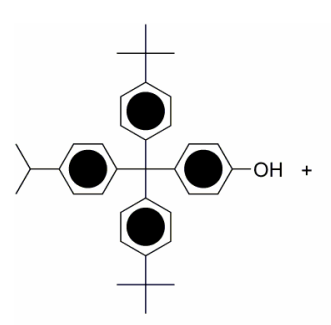

S2

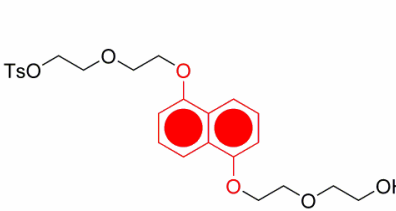

S3
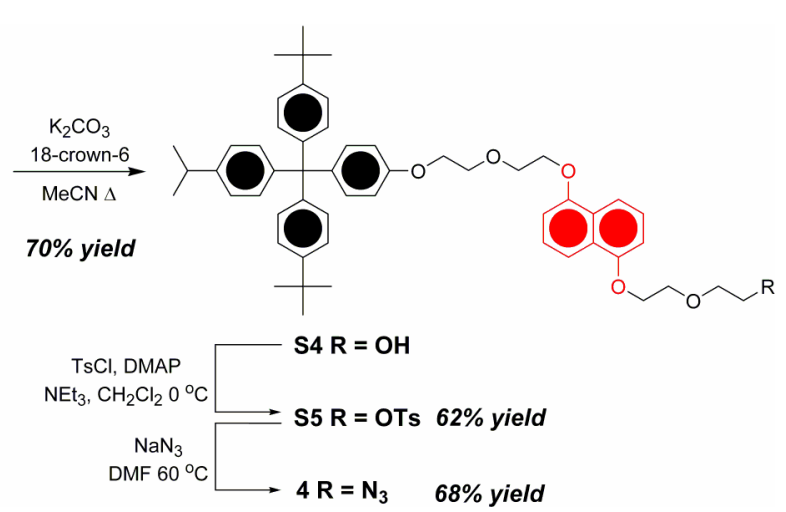

Preparation of Stopper-DNP-OH S4. Tetraarylmethane S2 (1.500 g, $3.057 \mathrm{mmol})$, dioxynaphthalene monotosylate derivative $\mathbf{S 3}(1.799 \mathrm{~g}, 3.668 \mathrm{mmol}){ }^{4} \mathrm{~K}_{2} \mathrm{CO}_{3}(1.690 \mathrm{~g}$, 
$12.23 \mathrm{mmol})$, and [18]crown-6 (0.040 g, $0.153 \mathrm{mmol})$ were taken up in $\mathrm{MeCN}(30 \mathrm{~mL})$. The heterogeneous solution was refluxed with vigorous stirring for $12 \mathrm{~h}$. The reaction mixture was filtered through celite and the solvent was evaporated. Flash chromatography of the crude product $\left(\mathrm{SiO}_{2}, 85: 15 \mathrm{CH}_{2} \mathrm{Cl}_{2}\right.$ : hexanes) yielded $1.725 \mathrm{~g}$ (70\% yield) of the alkylation product $\mathbf{S} 4$ as an amorphous white solid. ${ }^{1} \mathrm{H}$ NMR (500 $\left.\mathrm{MHz}, \mathrm{CDCl}_{3}, 25{ }^{\circ} \mathrm{C}, \mathrm{TMS}\right): \delta 7.88\left(\mathrm{~d},{ }^{3} \mathrm{~J}(\mathrm{H}, \mathrm{H})=9 \mathrm{~Hz}, 1 \mathrm{H}, \mathrm{DNP} p\right.$-O) $7.86\left(\mathrm{~d},{ }^{3} J(\mathrm{H}, \mathrm{H})\right.$ $=9 \mathrm{~Hz}, 1 \mathrm{H}, \mathrm{DNP}$ aryl $-\mathrm{H} p-\mathrm{O}), 7.32(\mathrm{~m}, 2 \mathrm{H}, \mathrm{DNP}$ aryl $-\mathrm{H} m-\mathrm{O}), 7.23(\mathrm{~m}, 4 \mathrm{H}$, stopper aryl $-\mathrm{H} o-\mathrm{tBu}), 7.09\left(\mathrm{~m}, 10 \mathrm{H}\right.$, stopper all meta Ar-H and $o$-iPr), $6.85\left(\mathrm{~d},{ }^{3} J(\mathrm{H}, \mathrm{H})=8 \mathrm{~Hz}\right.$, 1H, DNP aryl -H $o-\mathrm{O}), 6.84\left(\mathrm{~d},{ }^{3} J(\mathrm{H}, \mathrm{H})=8 \mathrm{~Hz}, 1 \mathrm{H}, \mathrm{DNP}\right.$ aryl $\left.-\mathrm{H} o-\mathrm{O}\right), 6.80\left(\mathrm{~d},{ }^{3} J(\mathrm{H}, \mathrm{H})\right.$ $=9 \mathrm{~Hz}, 2 \mathrm{H}$, stopper aryl $-\mathrm{H} o-\mathrm{O}), 4.31\left(\mathrm{~m}, 4 \mathrm{H}\right.$, both DNP-OCH $\left.\mathrm{OCH}_{2}\right), 4.16\left(\mathrm{t},{ }^{3} J(\mathrm{H}, \mathrm{H})=5\right.$ $\mathrm{Hz}, 2 \mathrm{H}$, stopper - $\left.\mathrm{OCH}_{2}\right), 4.07\left(\mathrm{t},{ }^{3} \mathrm{~J}(\mathrm{H}, \mathrm{H})=5 \mathrm{~Hz}, 2 \mathrm{H}\right.$, stopper $\left.-\mathrm{OCH}_{2} \mathrm{CH}_{2}\right), 4.00(\mathrm{~m}, 4 \mathrm{H}$, both DNP-OCH $\mathrm{CH}_{2}$ ), 3.78 (m, $2 \mathrm{H}, \mathrm{HOCH}_{2} \mathrm{CH}_{2}$ ), 3.75 (m, $2 \mathrm{H}, \mathrm{HOCH}$ ), 2.88 (septet, ${ }^{3} \mathrm{~J}$ $(\mathrm{H}, \mathrm{H})=7 \mathrm{~Hz}, 1 \mathrm{H}, \mathrm{iPr}-\mathrm{H}), 1.31(\mathrm{~s}, 18 \mathrm{H}, \mathrm{t}-\mathrm{Bu}), 1.24\left(\mathrm{~d},{ }^{3} \mathrm{~J}(\mathrm{H}, \mathrm{H})=7 \mathrm{~Hz}, 6 \mathrm{H}, \mathrm{iPr}-\mathrm{CH}_{3}\right)$; ${ }^{13} \mathrm{C}$ NMR $\left(125 \mathrm{MHz}, \mathrm{CDCl}_{3}, 25{ }^{\circ} \mathrm{C}\right): \delta 156.4,154.3,154.1,148.2,145.9,144.5,144.0$, 139.7, 132.1, 130.9, 130.6, 128.9, 128.8, 126.7, 126.6, 125.1, 125.1, 125.0, 124.0, 114.7, 114.4, 113.0, 105.7, 72.5, 70.0, 69.9, 69.7, 67.9, 67.8, 67.2, 63.0, 61.8, 34.2, 33.4, 23.9; HRMS (FAB): Calcd for $\mathrm{C}_{54} \mathrm{H}_{64} \mathrm{O}_{6} \mathrm{~m} / z=808.4703$. Found $m / z=808.4701$

Preparation of Stopper-DNP-OTs S5: S4 (0.300 g, 0371 mmol), DMAP (0.005 g, $0.037 \mathrm{mmol})$, and triethylamine $(0.261 \mathrm{~mL}, 1.854 \mathrm{mmol})$ were dissolved in $\mathrm{CH}_{2} \mathrm{Cl}_{2}(3.7$ $\mathrm{mL})$. The solution was cooled to $0{ }^{\circ} \mathrm{C}$, and $p$-toluenesulfonyl chloride $(0.085 \mathrm{~g}, 0.445$ $\mathrm{mmol}$ ) was added. The solution was allowed to warm slowly to RT while stirring for 12 h. The reaction mixture was diluted into $\mathrm{CH}_{2} \mathrm{Cl}_{2}(50 \mathrm{~mL})$, washed with $\mathrm{H}_{2} \mathrm{O}(2 \times 50 \mathrm{~mL})$, saturated $\mathrm{NH}_{4} \mathrm{Cl}(2 \times 50 \mathrm{~mL})$, brine $(1 \times 50 \mathrm{~mL})$, dried $\left(\mathrm{MgSO}_{4}\right)$, filtered, and the solvent evaporated. The crude product was passed through a short plug of $\mathrm{SiO}_{2}$ in $\mathrm{CH}_{2} \mathrm{Cl}_{2}$ to obtain $0.221 \mathrm{~g}\left(61.9 \%\right.$ yield) of the pure tosylated product S5. S5: ${ }^{1} \mathrm{H}$ NMR $(500 \mathrm{MHz}$, $\left.\mathrm{CDCl}_{3}, 25{ }^{\circ} \mathrm{C}, \mathrm{TMS}\right): \delta 7.83\left(\mathrm{~d},{ }^{3} J(\mathrm{H}, \mathrm{H})=9 \mathrm{~Hz}, 1 \mathrm{H}, \mathrm{DNP} p-\mathrm{O}\right), 7.79\left(\mathrm{~d},{ }^{3} J(\mathrm{H}, \mathrm{H})=9\right.$ $\mathrm{Hz}, 1 \mathrm{H}, \mathrm{DNP}$ aryl $-\mathrm{H} p-\mathrm{O}), 7.78\left(\mathrm{~d},{ }^{3} J(\mathrm{H}, \mathrm{H})=8 \mathrm{~Hz}, 2 \mathrm{H}\right.$, tosylate aryl $\left.-\mathrm{H} o-\mathrm{S}\right), 7.30(\mathrm{~m}$, 2H, DNP aryl $-\mathrm{H} m-\mathrm{O}), 7.23(\mathrm{~m}, 4 \mathrm{H}$, stopper aryl $-\mathrm{H} o-\mathrm{tBu}), 7.08(\mathrm{~m}, 10 \mathrm{H}$, stopper all meta Ar-H and $o$-iPr $), 6.85\left(\mathrm{~d},{ }^{3} J(\mathrm{H}, \mathrm{H})=8 \mathrm{~Hz}, 1 \mathrm{H}, \mathrm{DNP}\right.$ aryl $\left.-\mathrm{H} o-\mathrm{O}\right), 6.80\left(\mathrm{~d},{ }^{3} J(\mathrm{H}, \mathrm{H})\right.$ $=9 \mathrm{~Hz}, 2 \mathrm{H}$, stopper aryl $-\mathrm{H} o-\mathrm{O}), 6.78\left(\mathrm{~d},{ }^{3} J(\mathrm{H}, \mathrm{H})=8 \mathrm{~Hz}, 1 \mathrm{H}\right.$, DNP aryl $\left.-\mathrm{H} o-\mathrm{O}\right), 4.32$ 
$\left(\mathrm{t},{ }^{3} J(\mathrm{H}, \mathrm{H})=5 \mathrm{~Hz}, 2 \mathrm{H}\right), 4.19(\mathrm{~m}, 6 \mathrm{H}), 4.07\left(\mathrm{t},{ }^{3} J(\mathrm{H}, \mathrm{H})=5 \mathrm{~Hz}, 2 \mathrm{H}\right), 3.99\left(\mathrm{t},{ }^{3} J(\mathrm{H}, \mathrm{H})=5\right.$ $\mathrm{Hz}, 2 \mathrm{H}), 3.91\left(\mathrm{t},{ }^{3} J(\mathrm{H}, \mathrm{H})=5 \mathrm{~Hz}, 2 \mathrm{H}\right), 3.83\left(\mathrm{t},{ }^{3} J(\mathrm{H}, \mathrm{H})=5 \mathrm{~Hz}, 2 \mathrm{H}\right), 2.87$ (septet, ${ }^{3} J$ $(\mathrm{H}, \mathrm{H})=7 \mathrm{~Hz}, 1 \mathrm{H}, \mathrm{iPr}-\mathrm{H}), 2.35\left(\mathrm{~s}, 3 \mathrm{H}, \mathrm{OTs}-\mathrm{CH}_{3}\right), 1.29(\mathrm{~s}, 18 \mathrm{H}, \mathrm{t}-\mathrm{Bu}), 1.24\left(\mathrm{~d},{ }^{3} J(\mathrm{H}, \mathrm{H})\right.$ $\left.=7 \mathrm{~Hz}, 6 \mathrm{H}, \mathrm{iPr}-\mathrm{CH}_{3}\right) ;{ }^{13} \mathrm{C} \mathrm{NMR}\left(125 \mathrm{MHz}, \mathrm{CDCl}_{3}, 25^{\circ} \mathrm{C}\right): \delta 156.4,154.2,154.0,148.2$, $145.9,144.7,144.5,144.1,139.7,132.1,130.9,130.6,129.7,128.9,127.9,126.7,126.6$, 125.1, 125.0, 124.0, 114.7, 114.4, 113.0, 105.7, 105.6, 70.0, 69.8, 69.3, 68.9, 67.9, 67.8, 67.2, 63.0, 34.2, 33.3, 31.3, 29.6, 23.9, 21.5. HRMS (FAB): Calcd for $\mathrm{C}_{61} \mathrm{H}_{70} \mathrm{O}_{8} \mathrm{~S} \mathrm{~m} / \mathrm{z}=$ 962.4791. Found $\mathrm{m} / \mathrm{z}=962.4833$.

Preparation of Stopper-DNP-N3 (4): $\mathbf{S 5}(0.275 \mathrm{~g}, 0.285 \mathrm{mmol})$ and sodium azide $(0.186 \mathrm{~g}, 2.855 \mathrm{mmol})$ were stirred in DMF $(3.81 \mathrm{~mL})$ at $50{ }^{\circ} \mathrm{C}$ for $6 \mathrm{~h}$. The reaction mixture was filtered through celite and the solvent was evaporated. The resulting solid was sonicated in $\mathrm{CH}_{2} \mathrm{Cl}_{2}$ and soluble fractions chromatographed $\left(\mathrm{SiO}_{2}, 8: 2 \mathrm{CH}_{2} \mathrm{Cl}_{2}\right.$ eluent) to yield $0.170 \mathrm{~g}$ (68\% yield) of $\mathbf{4}$ as an amourphous white powder. 4: ${ }^{1} \mathrm{H}$ NMR (400 MHz, $\left.\mathrm{CDCl}_{3}, 25{ }^{\circ} \mathrm{C}, \mathrm{TMS}\right): \delta 7.89\left(\mathrm{~d},{ }^{3} J(\mathrm{H}, \mathrm{H})=8 \mathrm{~Hz}, 1 \mathrm{H}, \mathrm{DNP} p-\mathrm{O}\right), 7.87\left(\mathrm{~d},{ }^{3} J\right.$ $(\mathrm{H}, \mathrm{H})=8 \mathrm{~Hz}, 1 \mathrm{H}, \mathrm{DNP}$ aryl $-\mathrm{H} p-\mathrm{O}), 7.34(\mathrm{~m}, 2 \mathrm{H}, \mathrm{DNP}$ aryl $-\mathrm{H} m-\mathrm{O}), 7.23(\mathrm{~m}, 4 \mathrm{H}$, stopper aryl -H $o$-tBu), $7.09\left(\mathrm{~m}, 10 \mathrm{H}\right.$, stopper all meta Ar-H and $o$-iPr), $6.85\left(\mathrm{~d},{ }^{3} J(\mathrm{H}, \mathrm{H})\right.$ $=8 \mathrm{~Hz}, 1 \mathrm{H}, \mathrm{DNP}$ aryl $-\mathrm{H} o-\mathrm{O}), 6.84\left(\mathrm{~d},{ }^{3} J(\mathrm{H}, \mathrm{H})=8 \mathrm{~Hz}, 1 \mathrm{H}, \mathrm{DNP}\right.$ aryl $\left.-\mathrm{H} o-\mathrm{O}\right), 6.80(\mathrm{~d}$, ${ }^{3} J(\mathrm{H}, \mathrm{H})=9 \mathrm{~Hz}, 2 \mathrm{H}$, stopper aryl $\left.-\mathrm{H} o-\mathrm{O}\right), 4.32(\mathrm{~m}, 4 \mathrm{H}), 4.16\left(\mathrm{t},{ }^{3} J(\mathrm{H}, \mathrm{H})=5 \mathrm{~Hz}, 2 \mathrm{H}\right)$, $4.07\left(\mathrm{t},{ }^{3} J(\mathrm{H}, \mathrm{H})=5 \mathrm{~Hz}, 2 \mathrm{H}\right), 4.01(\mathrm{~m}, 4 \mathrm{H}), 3.84\left(\mathrm{t},{ }^{3} J(\mathrm{H}, \mathrm{H})=5 \mathrm{~Hz}, 2 \mathrm{H}\right), 3.44\left(\mathrm{t},{ }^{3} J\right.$ $\left.(\mathrm{H}, \mathrm{H})=5 \mathrm{~Hz}, 2 \mathrm{H},-\mathrm{CH}_{2} \mathrm{~N}_{3}\right), 2.88\left(\right.$ septet, $\left.{ }^{3} J(\mathrm{H}, \mathrm{H})=7 \mathrm{~Hz}, 1 \mathrm{H}, \mathrm{iPr}-\mathrm{H}\right), 1.30(\mathrm{~s}, 18 \mathrm{H}, \mathrm{t}-$ $\mathrm{Bu}), 1.24\left(\mathrm{~d},{ }^{3} J(\mathrm{H}, \mathrm{H})=7 \mathrm{~Hz}, 6 \mathrm{H}, \mathrm{iPr}-\mathrm{CH}_{3}\right) ;{ }^{13} \mathrm{C} \mathrm{NMR}\left(125 \mathrm{MHz}, \mathrm{CDCl}_{3}, 25{ }^{\circ} \mathrm{C}\right): \delta$ 156.5, 154.2, 154.1, 148.2, 145.9, 144.5, 144.0, 139.7, 132.1, 130.9, 130.6, 126.7, 126.6, 125.1, 125.1, 125.0, 124.0, 114.7, 114.5, 113.0, 105.6, 70.3, 70.0, 69.9, 69.8, 67.9, 67.2, 63.0, 50.7, 34.2, 33.3, 31.3, 23.9; HRMS (FAB): Calcd for $\mathrm{C}_{54} \mathrm{H}_{63} \mathrm{~N}_{3} \mathrm{O}_{5} \mathrm{~m} / z=873.4768$. Found $m / z=873.4736$. 
Scheme S4. Synthesis of dialkyne stopper derivative 5.

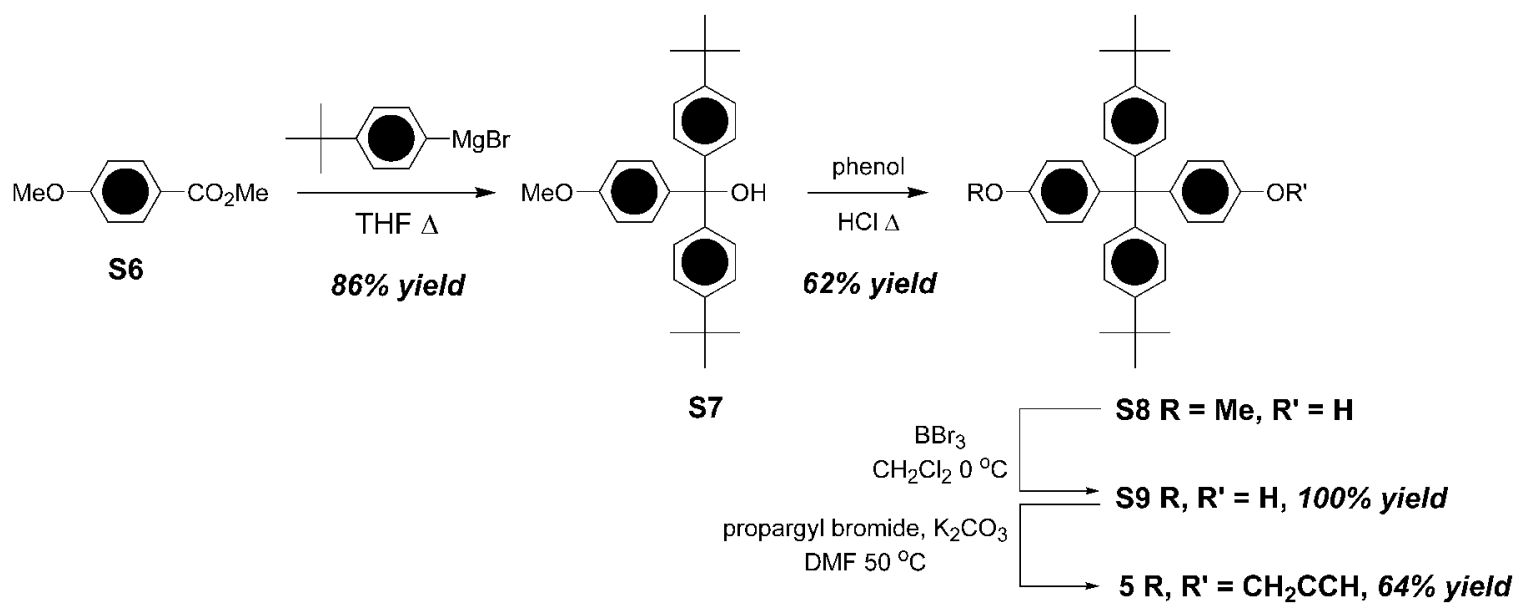

Preparation of Trityl Alcohol S7. 4-t-Butylphenyl bromide (24.5 mL, 30.1 g, 141.4 mmol) was added slowly to freshly cleaned magnesium turnings $(3.50 \mathrm{~g}, 144 \mathrm{mmol}$; cleaned by sonication in $\left.\mathrm{Et}_{2} \mathrm{O}\right)$ in $\mathrm{THF}(65 \mathrm{~mL})$ under an $\mathrm{Ar}$ atmosphere. The solution was heated under reflux for $4 \mathrm{~h}$ until the solid $\mathrm{Mg}$ had disappeared. The Grignard reagent was cooled down to $0{ }^{\circ} \mathrm{C}$ and a solution of methyl 4-methoxybenzoate (S6, $10.00 \mathrm{~g}, 60.2$ mmol) in the minimum amount of THF was added by cannula transfer. The mixture was heated under reflux for $1 \mathrm{~h}$, then was taken up in $\mathrm{CH}_{2} \mathrm{Cl}_{2}(100 \mathrm{~mL})$, washed with sat. $\mathrm{NH}_{4} \mathrm{Cl}(3 \times 100 \mathrm{~mL})$ and brine, dried $\left(\mathrm{MgSO}_{4}\right)$, and the solvent evaporated. The crude product was recrystallized from hexanes to yield $20.8 \mathrm{~g}$ (86\% yield) of $\mathbf{S 7}$ as white crystals. S7: ${ }^{1} \mathrm{H}$ NMR $\left(500 \mathrm{MHz}, \mathrm{CDCl}_{3}, 25{ }^{\circ} \mathrm{C}\right.$ ): $\delta$ 7.33-7.31 (m, $\left.4 \mathrm{H}, \mathrm{H}-\mathrm{Ar}\right), 7.21-7.18$ $(\mathrm{m}, 6 \mathrm{H}, \mathrm{H}-\mathrm{Ar}), 6.84\left(\mathrm{~d},{ }^{3} \mathrm{~J}(\mathrm{H}, \mathrm{H})=9 \mathrm{~Hz}, 2 \mathrm{H}, \mathrm{H}-\mathrm{Ar}\right), 3.80$ (s, $\left.3 \mathrm{H},-\mathrm{OCH}_{3}\right), 1.321$ (s, 18 $\left.\mathrm{H},-\mathrm{CH}_{3}\right) ;{ }^{13} \mathrm{C}$ NMR $\left(125 \mathrm{MHz}, \mathrm{CDCl}_{3}\right): \delta 158.4,149.8,144.1,139.5,129.0,127.4$, 124.6, 113.0, 81.3, 55.1, 34.3, 31.3; Anal. C: 83.74, H: 8.18 (calcd C: 83.54, H: 8.51). HRMS (FAB): Calcd for $\mathrm{C}_{28} \mathrm{H}_{34} \mathrm{O}_{2} \mathrm{~m} / z=402.2559$. Found $\mathrm{m} / z=402.2567$.

Preparation of Tetraarylmethane Alcohol S8. Trityl alcohol S7 (10.00 g, $24.2 \mathrm{mmol})$ and phenol $(45.4 \mathrm{~g}, 483 \mathrm{mmol})$ were mixed neat and heated to $80^{\circ} \mathrm{C}$ until the phenol melted. Concentrated $\mathrm{HCl}(1.1 \mathrm{~mL})$ was added and the solution was heated to $100^{\circ} \mathrm{C}$ for $5 \mathrm{~h}$. The reaction mixture was taken up in $\mathrm{PhMe}$, washed with aqueous $0.5 \mathrm{M} \mathrm{NaOH} \mathrm{(7} \mathrm{x}$ $75 \mathrm{~mL})$, and dried $\left(\mathrm{MgSO}_{4}\right)$, and the solvent evaporated to give the crude product as a yellow oil. Chromatography (silica gel, 25\% hexanes in $\mathrm{CH}_{2} \mathrm{Cl}_{2}$ ) afforded $6.06 \mathrm{~g}(62 \%$ 
yield) of S8 as a white powder. S8: ${ }^{1} \mathrm{H}$ NMR (400 MHz, $\left.\mathrm{CDCl}_{3}, 25^{\circ} \mathrm{C}\right): \delta$ 7.24-7.22 (m, 4 H, H-Ar), 7.10-7.04 (m, $8 \mathrm{H}, \mathrm{H}-\mathrm{Ar}), 6.77\left(\mathrm{~d},{ }^{3} J(\mathrm{H}, \mathrm{H})=9 \mathrm{~Hz}, 2 \mathrm{H}, \mathrm{H}-\mathrm{Ar}\right), 6.70\left(\mathrm{~d},{ }^{3} J\right.$ $(\mathrm{H}, \mathrm{H})=9 \mathrm{~Hz}, 2 \mathrm{H}, \mathrm{H}-\mathrm{Ar}), 4.63(\mathrm{~s}, 1 \mathrm{H},-\mathrm{OH}), 3.79\left(\mathrm{~s}, 3 \mathrm{H},-\mathrm{OCH}_{3}\right), 1.30\left(\mathrm{~s}, 18 \mathrm{H},-\mathrm{CH}_{3}\right)$; ${ }^{13} \mathrm{C}$ NMR $\left(100 \mathrm{MHz}, \mathrm{CDCl}_{3}\right): \delta 157.3,153.3,148.4,144.2,139.9,139.7,132.4,132.2$, 130.6, 129.1, 124.1, 114.0, 112.5, 62.8, 55.2, 34.3, 31.4; Anal. C: 84.89, H: 8.15 (calcd C: 85.31, H: 8.00). HRMS (FAB): Calcd for $\mathrm{C}_{34} \mathrm{H}_{38} \mathrm{O}_{2} \mathrm{~m} / \mathrm{z}=478.2872$. Found $\mathrm{m} / \mathrm{z}=$ 478.2886 .

Preparation of Tetraarylmethane Diol S9. A $1 \mathrm{M}$ solution of $\mathrm{BBr}_{3}$ in $\mathrm{CH}_{2} \mathrm{Cl}_{2}(23.0$ $\mathrm{mL}, 23.0 \mathrm{mmol}$ ) was added dropwise to a solution of methyl aryl ether $\mathbf{S 8}(5.00 \mathrm{~g}, 10.4$ mmol) in $\mathrm{CH}_{2} \mathrm{Cl}_{2}(350 \mathrm{~mL})$ at $0{ }^{\circ} \mathrm{C}$. The reaction mixture was warmed up to room temperature and stirred for $36 \mathrm{~h}$ under $\mathrm{Ar}$. $\mathrm{MeOH}(5 \mathrm{~mL})$ and then $\mathrm{H}_{2} \mathrm{O}(200 \mathrm{~mL})$ were added to quench the reaction. The organic layer was separated and collected. The aqueous layer was washed with $\mathrm{CH}_{2} \mathrm{Cl}_{2}(3 \times 200 \mathrm{~mL})$ and the combined organic layers were dried $\left(\mathrm{MgSO}_{4}\right)$. Removal of the solvent in vacuo afforded $4.85 \mathrm{~g}$ (100\% yield) of S9 as an orange powder requiring no further purification. S9: ${ }^{1} \mathrm{H}$ NMR $(500 \mathrm{MHz}$, $\left.\mathrm{CDCl}_{3}, 25{ }^{\circ} \mathrm{C}\right): \delta 7.23\left(\mathrm{~d},{ }^{3} J(\mathrm{H}, \mathrm{H})=9 \mathrm{~Hz}, 4 \mathrm{H}, \mathrm{H}-\mathrm{Ar}\right), 7.07\left(\mathrm{~d},{ }^{3} J(\mathrm{H}, \mathrm{H})=9 \mathrm{~Hz}, 4 \mathrm{H}, \mathrm{H}-\right.$ $\mathrm{Ar}), 7.04\left(\mathrm{~d},{ }^{3} \mathrm{~J}(\mathrm{H}, \mathrm{H})=9 \mathrm{~Hz}, 4 \mathrm{H}, \mathrm{H}-\mathrm{Ar}\right), 6.70\left(\mathrm{~d},{ }^{3} \mathrm{~J}(\mathrm{H}, \mathrm{H})=9 \mathrm{~Hz}, 4 \mathrm{H}, \mathrm{H}-\mathrm{Ar}\right), 1.30(\mathrm{~s}$, $\left.18 \mathrm{H},-\mathrm{CH}_{3}\right) ;{ }^{13} \mathrm{C} \mathrm{NMR}\left(125 \mathrm{MHz}, \mathrm{CDCl}_{3}\right): \delta 153.1,148.3,144.0,139.8,132.3,130.5$, 124.0, 113.9, 62.7, 34.2, 31.3; HRMS (FAB): Calcd for $\mathrm{C}_{33} \mathrm{H}_{36} \mathrm{O}_{2} \mathrm{~m} / z=464.2715$. Found $m / z=464.2721$.

Preparation of Diacetylene 5. Propargyl bromide ( $80 \% \mathrm{w} / \mathrm{w}$ solution in xylenes, $4.80 \mathrm{~g}$, $32.3 \mathrm{mmol})$ and $\mathrm{K}_{2} \mathrm{CO}_{3}(2.68 \mathrm{~g}, 19.4 \mathrm{mmol})$ were added to a solution of diol $\mathbf{S 9}(1.50 \mathrm{~g}$, $3.23 \mathrm{mmol})$ in DMF $(20 \mathrm{~mL})$ was added. The reaction mixture was heated to $50{ }^{\circ} \mathrm{C}$ and stirred for $40 \mathrm{~h}$ under Ar. The reaction was taken up in EtOAc $(150 \mathrm{~mL})$, washed with 1 $\mathrm{M} \mathrm{NaHSO}_{4}(1 \times 150 \mathrm{~mL}), \mathrm{H}_{2} \mathrm{O}(2 \times 100 \mathrm{~mL})$, and brine $(2 \times 100 \mathrm{~mL})$, dried $\left(\mathrm{MgSO}_{4}\right)$, and evaporated onto silica gel. Chromatography (silica gel, 10\% EtOAc in hexanes) afforded $1.12 \mathrm{~g}$ (64\% yield) of 5. 5: ${ }^{1} \mathrm{H}$ NMR $\left(500 \mathrm{MHz}, \mathrm{CDCl}_{3}, 25{ }^{\circ} \mathrm{C}\right): \delta 7.24\left(\mathrm{~d},{ }^{3} J\right.$ $(\mathrm{H}, \mathrm{H})=9 \mathrm{~Hz}, 4 \mathrm{H}, \mathrm{H}-\mathrm{Ar}), 7.10\left(\mathrm{~d},{ }^{3} J(\mathrm{H}, \mathrm{H})=9 \mathrm{~Hz}, 4 \mathrm{H}, \mathrm{H}-\mathrm{Ar}\right), 7.07\left(\mathrm{~d},{ }^{3} J(\mathrm{H}, \mathrm{H})=9\right.$ $\mathrm{Hz}, 4 \mathrm{H}, \mathrm{H}-\mathrm{Ar}), 6.85\left(\mathrm{~d},{ }^{3} J(\mathrm{H}, \mathrm{H})=9 \mathrm{~Hz}, 4 \mathrm{H}, \mathrm{H}-\mathrm{Ar}\right), 4.66\left(\mathrm{~d},{ }^{4} J(\mathrm{H}, \mathrm{H})=2 \mathrm{~Hz}, 4 \mathrm{H}\right.$, - 
$\left.\mathrm{CH}_{2^{-}}\right), 2.52\left(\mathrm{t},{ }^{4} \mathrm{~J}(\mathrm{H}, \mathrm{H})=2 \mathrm{~Hz}, 2 \mathrm{H}, \mathrm{H}\right.$-acetylene), $1.30\left(\mathrm{~s}, 18 \mathrm{H},-\mathrm{CH}_{3}\right) ;{ }^{13} \mathrm{C}$ NMR $(125$ $\left.\mathrm{MHz}, \mathrm{CDCl}_{3}\right): \delta 155.4,148.3,143.9,140.3,132.0,130.5,124.0,113.3,78.7,75.3,62.7$, 55.7, 34.2, 31.3; HRMS (FAB): Calcd for $\mathrm{C}_{39} \mathrm{H}_{40} \mathrm{O}_{2} \mathrm{~m} / \mathrm{z}=540.3028$. Found $\mathrm{m} / \mathrm{z}=$ 540.3042 .

\section{References:}

(1) Rowan, S. J.; Stoddart, J. F. Org. Lett. 1999, 1, 1913.

(2) Asakawa, M.; Dehaen, W.; Labbe, G.; Menzer, S.; Nouwen, J.; Raymo, F. M.; Stoddart, J. F.; Williams, D. J. J. Org. Chem. 1996, 61, 9591.

(3) Ashton, P. R.; Ballardini, R.; Balzani, V.; Belohradsky, M.; Gandolfi, M. T.; Philp, D.; Prodi, L.; Raymo, F. M.; Reddington, M. V.; Spencer, N.; Stoddart, J. F.; Venturi, M.; Williams, D. J. J. Am. Chem. Soc. 1996, 118, 4931.

(4) Jeppesen, J. O.; Perkins, J.; Becher, J.; Stoddart, J. F. Angew. Chem. Int. Ed. 2001, 40, 1216.

(5) Simpson, C. D.; Mattersteig, G.; Martin, K.; Gherghel, L.; Bauer, R. E.; Rader, H. J.; Müllen, K. J. Am. Chem. Soc. 2004, 126, 3139.

\section{Full References from the Manuscript:}

(1a) Choi, J. W.; Flood, A. H.; Steuerman, D.; Nygaard, S.; Braunschweig, A. B.; Moonen, N. N. P.; Laursen, B. W.; Luo, Y.; Delonno, E.; Peters, A. J.; Jeppesen, J. O.; Xu, K.; Stoddart, J. F.; Heath, J. R. Chem. Eur. J. 2006, 12, 261.

(1e) Luo, Y.; Collier, C. P.; Jeppesen, J. O.; Nielsen, K. A.; DeIonno, E.; Ho, G.; Perkins, J.; Tseng, H. R.; Yamamoto, T.; Stoddart, J. F.; Heath, J. R. ChemPhysChem 2002, 3, 519.

(11) Liu, Y.; Flood, A. H.; Bonvallett, P. A.; Vignon, S. A.; Northrop, B. H.; Tseng, H. R.; Jeppesen, J. O.; Huang, T. J.; Brough, B.; Baller, M.; Magonov, S.; Solares, S. D.; Goddard, W. A.; Ho, C. M.; Stoddart, J. F. J. Am. Chem. Soc. 2005, 127, 9745. 